\title{
Qualidade de vida e autoestima de pacientes com úlcera venosa
}

RESUMO | Objetivo: Avaliar a qualidade de vida e autoestima de pacientes com úlcera venosa. Método: Estudo primário, exploratório, observacional, transversal e descritivo realizado com 75 pacientes atendidos na Atenção Primária de Conselheiro Lafaiete-MG no período de dezembro de 2018 a maio de 2019 com a aplicação da Escala de autoestima de Rosenberg e o questionário de qualidade de vida SF-36. Resultados: O gênero feminino foi mais prevalente (60\%), idade média de 68,9 anos e predomínio de doenças crônico-degenerativas. O levantamento das médias da Escala de Autoestima de Rosenberg teve maiores pontuações foram relacionadas ao gênero feminino, raça branca, aposentados, tabagistas, etilistas, com HAS e DM. Conclusão: A presença de úlcera venosa interferiu negativamente na autoestima de ambos os gêneros, entretanto o gênero masculino apresenta melhores escores quando comparados ao gênero feminino.

Palavras-chaves: Úlcera Varicosa; Estomaterapia; Cuidados de Enfermagem; Ferimentos e Lesões; Saúde da Família.

ABSTRACT | To assess the quality of life and self-esteem of patients suffering from venous ulcer. Method: Cross-sectional study regarding 75 patients, seen in the family health units in the Conselheiro Lafaiete municipality-MG. The individual basic care registration form, Rosenberg's self-esteem scale and the SF-36 quality of life survey were utilized for the data gathering. Results: Attested female predominance(60\%), with a median age of 68,9 years. The Rosenberg's self-esteem scale attested an average of 9,7, the greatest average of the SF-36 test was 57,7. The Overall Health Status variable indicated the highest score among those evaluated, correlating to improvement, while the Physical Aspect corresponded to worsening, especially when associated with the female sex and the presence of comorbid conditions. Conclusion: The presence of venous ulcers interfered the self-esteem of both genders.

Keywords: Varicose Ulcer; Stomatherapy; Nursing Care; Wounds And Injuries; Family Health. Mily Health.

RESUMEN I Objetivo: Evaluar la calidad de vida y la autoestima de pacientes con úlceras venosas. Método: Estudio primario, exploratorio, observacional, transversal y descriptivo realizado con 75 pacientes atendidos en la Atención Primaria de Conselheiro Lafaiete-MG entre diciembre 2018 y mayo 2019 con la aplicación de la escala de autoestima de Rosenberg y el cuestionario de calidad de vida SF-36. Resultados: el sexo femenino tuvo mayor prevalencia (60\%), con edad promedia de 68,9 años y predominio de enfermedades crónico-degenerativas. Los promedios de la escala de Rosenberg con mayor puntuación fueron los relacionados al género femenino, de raza blanca, jubilados, fumadores, usuarios de bebidas alcohólicas, con HSA y DM. Conclusión: la presencia de úlceras venosas interfirió negativamente en la autoestima de ambos sexos, sin embargo, el sexo masculino presenta mejores puntajes cuando comparados al sexo femenino.

Palabras claves: Úlcera varicosa; Estomaterapia; Cuidados de enfermeria; Heridas y traumatismos; Salud de la familia.

\section{Hélio Martins do Nascimento Filho}

Enfermeiro. Professor. Revisor da Revista Científica Avances en Enfermería. Graduação em Enfermagem, Universidade Presidente Antônio Carlos (2010). Mestre em Ciência, Tecnologia e Gestão Aplicadas à Regeneração Tecidual pela Universidade Federal de São Paulo/SP - Unifesp.

ORCID: 0000-0003-3700-3910

\section{Leila Blanes}

Enfermeira. Professora. Graduação em Enfermagem, de Enfermagem pela Universidade Federal de São Paulo (1996). Doutorado (2005) em Ciências pelo Programa de Pós-Graduação em Cirurgia Plástica da Universidade Federal de São Paulo- UNIFESP.

ORCID: 0000-0002-6922-7719

\section{Natália Ferreira Gonçalves P. de Castro}

Acadêmica de enfermagem pela Faculdade Santa Rita- FaSaR- Conselheiro Lafaiete - MG (2015); voluntária no Projeto "Viver sem feridas"; Pesquisadora do projeto: Qualidade de vida dos usuários com úlcera venosa cadastrado nas Equipes de Estratégia Saúde da Família do município de Conselheiro Lafaiete- Minas Gerais pela UNIFESP (2018).

ORCID: 0000-0003-3720-2477

\section{Beatriz Maia Prado}

Academica da Escola Paulista de Enfermagem da Universidade Federal de São Paulo (EPE - UNIFESP). Membro associado da Sociedade de Cardiologia do Estado de São Paulo (SOCESP). Atual bolsista no Programa Institucional de Bolsas de Extensão da Unifesp (PIBEX) pelo projeto de extensão Recreação para idosos hospitalizados. ORCID: 0000-0002-1319-8082

\section{Daniela Tinti Moreira Borges}

Enfermeira. Professora. Graduada em Enfermagem pela Universidade Federal de Mato Grosso do Sul (2006). Mestre em Ciência, Tecnologia e Gestão Aplicadas à Regeneração Tecidual pela Universidade Federal de São Paulo/SP.

ORCID: 0000-0001-8890-8918

\section{Flávia Carla Takaki Cavichioli}

Enfermeira. Graduada em Enfermagem pela Universidade De Mato Grosso do Sul (2006) e Bacharel em Administração pelo Instituto Educacional do Estado de São Paulo (2015). Mestre em Ciência, Tecnologia e Gestão Aplicadas a Regeneração Tecidual pela UNIFESP. ORCID: 0000-0003-2277-9548

\section{Lydia Masako Ferreira}

Médica. Professora. Graduação em Medicina na Universidade de Mogi das Cruzes (1976). Pós-Doutorado University of California, San FrancisCo (1992), Livre Docência UNIFESP (1994), ORCID: 0000-0003-4587-509X 
INTRODUÇÃO

$\mathrm{O}$ crescimento da expectativa de vida resultou no aumento da ocorrência de doenças crônicas, entre elas, a Ulcera Crônica, que atinge $1 \%$ da população adulta. A Ulcera Venosa de membros inferiores representa 70 a 90\% dos casos das Úlceras Crônicas e ocorre devido a Insuficiência Venosa Crônica (IVC) e por traumas no terço inferior das pernas, principalmente nas regiões maleolares medial e lateral ${ }^{(1-8)}$.

De acordo com a Scottish Intercollegiate Guidelines Network, as Úlceras Venosas também denominada úlcera de estase ou varicosa, são as lesões mais graves da Síndrome da Insuficiência Venosa Crônica dos membros inferiores. A úlcera Venosa é um problema de saúde pública por demandar cuidados contínuos, gerar altos custos para os sistemas de saúde e restringir a capacidade funcional do indivíduo interferindo de forma negativa na qualidade de vida dos pacientes ${ }^{(2-13)}$.

Nos Estados Unidos da América, cerca de um bilhão de dólares são gastos anualmente com o tratamento de pacientes com a ferida. No Reino Unido o valor gasto com esses pacientes chega a quase $£ 200$ milhões por ano. Em países ocidentais, os estudos realizados demonstram que a estimativa da prevalência da UV é de 1,0\% na população geral e 2,0\% nos idosos com 80 anos ou mais. A úlcera Venosa apresenta recidiva de quase $80 \%$ no primeiro ano de cicatrização e cerca de 1,0\% dos recursos destinados a tratamento de saúde são gastos com tratamento dos doentes $(1,3,5,9,14)$.

Cerca de 3\% da população brasileira apresenta Ulcera Venosa, de modo que ela é a $14^{\text {a }}$ causa de afastamento de atividades laborais no país e a $34^{\mathrm{a}}$ causa de aposentadorias;

tal fato pode estar relacionado à baixa taxa de adesão à terapia compressiva. Estudos científicos retratam a dificuldade de adesão ao tratamento por parte do paciente, falta de adoção de hábitos alimentares saudáveis para o controle do
A Úlcera Venosa é de difícil manejo clínico e sua cicatrização ocorre por segunda intenção. Além dos sinais e sintomas físicos (dor, prurido, odor desagradável e prejuízo da mobilidade), queixas psicológicas como ansiedade, depressão, perturbação do sono, isolamento social, solidão e perda da independência são relatos comuns de pacientes com UV e pode interferir na Qualidade de Vida (QV). peso, pouca prática de exercício físico e repouso. A baixa adesão devido ao longo tempo de tratamento e constantes trocas de curativo pode implicar na manutenção da lesão e na elevada taxa de recidiva da ferida de $62,2 \%$ dos $\operatorname{casos}^{(4,7,8,13,15)}$.

A Úlcera Venosa é de difícil manejo clínico e sua cicatrização ocorre por segunda intenção. Além dos sinais e sintomas físicos (dor, prurido, odor desagradável e prejuízo da mobilidade), queixas psicológicas como ansiedade, depressão, perturbação do sono, isolamento social, solidão e perda da independência são relatos comuns de pacientes com UV e pode interferir na Qualidade de Vida (QV). A presença da lesão provoca alteração nas atividades diárias e de trabalho, alterações na autoimagem e na capacidade de desempenhar a atividade laboral, estimulando o isolamento social ${ }^{(1,2,3,4,57,8,9,13,14)}$.

Em Conselheiro Lafaiete, cidade de médio porte do interior de Minas Gerais, localizada a cerca de 100 quilômetros da capital Belo Horizonte, a prevalência de feridas crônicas em pacientes atendidos nas Unidades de Saúde da Família (USF) é de $0,164 \%$ e $50 \%$ das úlceras tratadas nas USF possuem etiologia venosa ${ }^{(16)}$.

O objetivo do estudo foi avaliar a qualidade de vida e a autoestima de pacientes com úlcera venosa atendidos nas Unidades de Saúde da Família no Município de Conselheiro Lafaiete - Minas Gerais.

\section{MÉTODO}

A pesquisa foi aprovada pelo Comitê de Ética em Pesquisa (CEP) da Universidade Federal de São Paulo - Unifesp com CAAE 00462818.2.0000.5505.

Trata-se de um estudo primário, exploratório, observacional, transversal e com análise descritiva dos resultados, realizado na Atenção Primária de ConseIheiro Lafaiete-MG, cidade com 116.000 habitantes, composta por 28 equipes de Unidades Saúde de Família (USF) e 06 Postos de Saúde responsáveis pela cober- 
tura de $87,05 \%$ da população de acordo com o Departamento de Atenção Básica.

A pesquisa teve amostra de 90 pacientes identificados durante o período de coleta dos dados e 75 destes aceitaram participar do estudo. Todos tinham idade acima dos 18 anos. A coleta dos dados ocorreu de dezembro de 2018 a maio de 2019 e foram utilizados os seguintes instrumentos: ficha de cadastro individual da Atenção Básica, Escala de autoestima de Rosenberg e o questionário de qualidade de vida SF-36.

Tabela 1: Pontuação para domínios do SF-36 e Escala de Autoestima de Rosenberg em pacientes com úlcera venosa em Conselheiro Lafaiete/MG (N=75) 2019

\begin{tabular}{lccccc}
\multicolumn{1}{c}{ Instrumentos } & Média & Mediana & Desvio Padrão & Min & Max \\
\hline SF-36 & & & & & \\
\hline Capacidade Funcional & 37,9 & 30 & 29,6 & 0 & 100 \\
\hline Aspecto Físico & 26,3 & 0 & 37,6 & 0 & 100 \\
\hline Dor & 49,1 & 50 & 29,1 & 0 & 100 \\
\hline Estado Geral de Saúde & 57,7 & 55 & 17,2 & 25 & 95 \\
\hline Vitalidade & 52,9 & 55 & 13,6 & 20 & 95 \\
\hline Aspecto Social & 33,8 & 37,5 & 20,9 & 0 & 87,5 \\
\hline Aspecto Emocional & 33,3 & 0 & 42,1 & 0 & 100 \\
\hline Saúde Mental & 54,1 & 56 & 11,2 & 28 & 80 \\
\hline Escala de Autoestima de Rosenberg & 9,7 & 9 & 5,8 & 0 & 22 \\
Fonte: autor, 2020 & & & & &
\end{tabular}

Tabela 2: Pontuação do SF-36 e Rosenberg segundo o gênero em pacientes com úlcera venosa em Conselheiro Lafaiete -2019.

\begin{tabular}{lccccc}
\multicolumn{1}{c}{ Instrumentos } & \multicolumn{2}{c}{ Feminino } & \multicolumn{2}{c}{ Masculino } & \\
& Média (DP) & Mín.-Máx. & Média (DP) & Mín.-Máx. & p-valor \\
SF-36 & & & & & \\
\hline Capac. Funcional & $35,00(26,99)$ & $0-90$ & $42,33(33,03)$ & $0-100$ & 0,296 \\
\hline Aspecto físico & $22,22(34,63)$ & $0-100$ & $32,50(41,60)$ & $0-100$ & 0,249 \\
\hline Dor & $50,67(28,48)$ & $0-100$ & $46,67(30,32)$ & $0-100$ & 0,563 \\
\hline Estado Geral de Saúde & $59,00(15,80)$ & $30-95$ & $55,67(19,11)$ & $25-90$ & 0,413 \\
\hline Vitalidade & $51,00(11,95)$ & $20-70$ & $55,67(15,52)$ & $30-95$ & 0,146 \\
\hline Aspecto Social & $34,72(20,62)$ & $0-75$ & $32,50(21,68)$ & $0-87,5$ & 0,656 \\
\hline Aspecto Emocional & $32,59(41,13)$ & $0-100$ & $34,44(44,19)$ & $0-100$ & 0,853 \\
\hline Saúde Mental & $53,60(11,61)$ & $28-80$ & $54,93(10,76)$ & $32-76$ & 0,618 \\
\hline Rosenberg & $9,80(5,22)$ & $0-21$ & $9,67(6,61)$ & $0-22$ & 0,923 \\
(DP) = Desvio padrão & & & & & \\
Fonte: autor, 2020 & & & & &
\end{tabular}

O SF-36 é constituído por 36 perguntas, que são agrupadas em oito domínios. As pontuações variam de 0 a 100 , onde 0 $=$ pior e $100=$ melhor, pontuações mais altas indicam melhor estado de saúde ${ }^{(17)}$.

Para a análise estatística foram utilizados o Teste de Igualdade de Duas Proporções, o Teste de Qui-Quadrado para Independência, o Teste de ANOVA (Analysis of variance), a Correlação de Pearson e o Intervalo de Confiança para a Média.

\section{RESULTADOS}

Dentre os 75 participantes da pesquisa, predominaram o gênero feminino (60\%), com média de idade de 68,9 anos (min. 38 e máx. 94), 57,3\% se autodeclararam pardos, $26,7 \%$ negros e $16 \%$ brancos. Em relação à situação no mercado de trabalho, $72 \%$ eram aposentados/pensionistas, $17,3 \%$ não trabalhavam, 6,7\% trabalhavam e 2,7\% estavam desempregados. Dentre as doenças preexistentes, 73,35\% apresentaram diagnóstico de hipertensão e $32 \%$ diabetes mellitus. Etilistas eram $6,7 \%$ e tabagistas $14,7 \%$.

Para a Escala de Autoestima de Rosenberg, a média foi de 9,7 (min. 0 e máx. 22). A Tabela 1 mostra a distribuição da média dos escores para cada domínio do SF-36. A maior média e o desvio padrão foi de $57,7(17,2)$ para Estado Geral de Saúde, seguido por Vitalidade com média e desvio padrão de 52,9 $(13,6)$. A menor média e desvio padrão foi de $26,3(37,6)$ para o domínio Aspecto Físico.

A comparação dos escores do SF-36 de acordo com o gênero é apresentada na Tabela 2. Para cinco dos oito domínios (Capacidade Funcional, Aspecto Físico, Vitalidade, Aspecto Emocional e Saúde Mental) o gênero masculino apresentou maiores pontuações que o feminino, e para os domínios Dor, Estado Geral de Saúde e Aspecto Social, o gênero feminino apresentou pontuações maiores que o masculino. Para a Escala de Autoestima de Rosenberg, a média foi de 9,80 (min. 0 e máx. 21) para o gênero feminino e 9,67 (min. 0 e máx. 22) para o gênero mascu- 
lino. Embora haja diferenças nos escores entre os gêneros, elas não apresentaram diferença estatística.

A Tabela 3 apresenta a pontuação nos escores da escala de autoestima de Ro- senberg e SF-36 em relação à presença de Hipertensão Arterial Sistêmica (HAS) e Diabetes Mellitus (DM). O valor médio seguido do desvio padrão para a escala de Rosenberg foi maior nos pacientes com DM,
Tabela 2: Pontuação do SF-36 e Rosenberg segundo o gênero em pacientes com úlcera venosa em Conselheiro Lafaiete -2019.

\begin{tabular}{|c|c|c|c|c|c|c|c|}
\hline \multirow{2}{*}{ Instrumentos } & & \multicolumn{2}{|c|}{ DM } & \multicolumn{4}{|c|}{ HAS } \\
\hline & & $\begin{array}{l}\text { Média } \\
\text { (DP) }\end{array}$ & Min-Máx & $\begin{array}{l}\text { p-va- } \\
\text { lor }\end{array}$ & $\begin{array}{l}\text { Média } \\
\text { (DP) }\end{array}$ & Min-Máx & $\begin{array}{l}\text { p-va- } \\
\text { lor }\end{array}$ \\
\hline \multicolumn{8}{|l|}{ SF-36 } \\
\hline \multirow[t]{2}{*}{ Capac. Funcional } & Não & $\begin{array}{c}33,73 \\
(27,03)\end{array}$ & $0-85$ & 0,072 & $\begin{array}{c}36,00 \\
(31,69)\end{array}$ & $0-90$ & 0,735 \\
\hline & Sim & $\begin{array}{c}46,88 \\
(33,16)\end{array}$ & $0-100$ & & $\begin{array}{c}38,64 \\
(29,02)\end{array}$ & $0-100$ & \\
\hline \multirow[t]{2}{*}{ Aspecto físico } & Não & $\begin{array}{c}27,45 \\
(38,16)\end{array}$ & $0-100$ & 0,711 & $\begin{array}{c}22,50 \\
(40,47)\end{array}$ & $0-100$ & 0,598 \\
\hline & Sim & $\begin{array}{c}23,96 \\
(37,21)\end{array}$ & $0-100$ & & $\begin{array}{c}27,73 \\
(36,85)\end{array}$ & $0-100$ & \\
\hline \multirow[t]{2}{*}{ Dor } & Não & $\begin{array}{c}51,57 \\
(29,21)\end{array}$ & $0-100$ & 0,281 & $\begin{array}{c}50,50 \\
(34,86)\end{array}$ & $0-100$ & 0,799 \\
\hline & Sim & $\begin{array}{c}43,75 \\
(28,71)\end{array}$ & $0-90$ & & $\begin{array}{c}48,55 \\
(27,04)\end{array}$ & $0-100$ & \\
\hline \multirow[t]{2}{*}{$\begin{array}{l}\text { Estado Geral de } \\
\text { Saúde }\end{array}$} & Não & $\begin{array}{c}57,25 \\
(17,59)\end{array}$ & $25-95$ & 0,764 & $\begin{array}{c}54,75 \\
(16,26)\end{array}$ & $25-80$ & 0,378 \\
\hline & Sim & $\begin{array}{c}58,54 \\
(16,52)\end{array}$ & $30-85$ & & $\begin{array}{c}58,73 \\
(17,49)\end{array}$ & $25-95$ & \\
\hline \multirow[t]{2}{*}{ Vitalidade } & Não & $\begin{array}{c}52,16 \\
(14,36)\end{array}$ & $20-95$ & 0,513 & $\begin{array}{c}56,25 \\
(13,46)\end{array}$ & $30-95$ & 0,195 \\
\hline & Sim & $\begin{array}{c}54,38 \\
(11,92)\end{array}$ & $30-85$ & & $\begin{array}{c}51,64 \\
(13,54)\end{array}$ & $20-85$ & \\
\hline \multirow[t]{2}{*}{ Aspecto Social } & Não & $\begin{array}{c}33,33 \\
(21,89)\end{array}$ & $0-87,5$ & 0,765 & $\begin{array}{c}35,00 \\
(20,52)\end{array}$ & $0-62,5$ & 0,773 \\
\hline & Sim & $\begin{array}{c}34,90 \\
(19,15)\end{array}$ & $0-62,5$ & & $\begin{array}{c}33,41 \\
(21,25)\end{array}$ & $0-87,5$ & \\
\hline \multirow[t]{2}{*}{$\begin{array}{l}\text { Aspecto Emo- } \\
\text { cional }\end{array}$} & Não & $\begin{array}{c}33,99 \\
(41,89)\end{array}$ & $0-100$ & 0,846 & $\begin{array}{c}30,00 \\
(44,46)\end{array}$ & $0-100$ & 0,682 \\
\hline & Sim & $\begin{array}{c}31,94 \\
(43,38)\end{array}$ & $0-100$ & & $\begin{array}{c}34,55 \\
(41,56)\end{array}$ & $0-100$ & \\
\hline \multirow[t]{2}{*}{ Saúde Mental } & Não & $\begin{array}{c}52,31 \\
(10,82)\end{array}$ & $28-80$ & 0,040 & $\begin{array}{l}56,20 \\
(8,65)\end{array}$ & $36-72$ & 0,340 \\
\hline & Sim & $\begin{array}{c}58,00 \\
(11,31)\end{array}$ & $28-76$ & & $\begin{array}{c}53,38 \\
(12,01)\end{array}$ & $28-80$ & \\
\hline Rosenberg & Não & $\begin{array}{c}9,49 \\
(5,70)\end{array}$ & $0-22$ & 0,578 & $9,40(6,32)$ & $0-22$ & 0,756 \\
\hline
\end{tabular}

(DP) $=$ Desvio padrão

Fonte: autor, 2020 com pontuação 10,29 $(6,00)$ e HAS com pontuação de 9,87 $(5,61)$. Em pacientes com DM foram apresentadas maiores médias em quatro dos oito domínios: Capacidade Funcional $(46,88)$, Estado Geral de Saúde $(58,54)$, Vitalidade $(54,38)$, Aspecto Social $(34,90)$ e Saúde Mental $(58,00)$. Nos pacientes com HAS a média das pontuações foram em quatro domínios, dois em comum aos diagnosticados com DM: Capacidade Funcional $(38,64)$, Estado Geral de Saúde $(58,73)$, Aspecto Físico $(27,73)$ e Aspecto Emocional $(34,55)$.

Houve diferença estatística em relação ao escore do SF-36 no domínio Saúde Mental na análise de DM, onde pessoas sem DM tiveram média de 52,31 em relação a 58,00 de média para pessoas com DM (p-valor = 0,040).

Em relação aos hábitos de vida como etilismo e tabagismo, a Escala de Autoestima de Rosenberg apresentou maiores médias para tabagistas $(10,91)$ e etilistas $(12,80)$. Nos domínios da SF-36 foram descritos maiores médias nos domínios Capacidade Funcional $(38,59)$, Vitalidade $(52,89)$ e Saúde Mental $(54,44)$ em pacientes não tabagistas. Em pacientes que não são etilistas, as maiores médias foram nos domínios Capacidade Funcional $(38,14)$, Aspecto Físico $(26,43)$, Aspecto Social $(34,11)$, Aspecto Emocional $(33,81)$ e Saúde Mental $(54,29)$.

\section{DISCUSSÃO}

Os limites dos resultados deste estudo referem-se ao delineamento transversal que não possibilita o estabelecimento de relações de causa e efeito, mas apontam associações importantes.

Na caracterização da população, em relação à idade, observou-se média de 68,9 anos, corroborando com estudos que apontam predomínio de pessoas acima de 60 anos com úlcera venosa ${ }^{(18,13)}$, o que confirma o fato de que com o aumento da expectativa de vida, o predomínio de doenças crônico-degenerativas e suas complicações, são desafios tanto para a sociedade quanto o sistema de saúde ${ }^{(16,8)}$. 
Através do levantamento das médias da Escala de Autoestima de Rosenberg foi possível observar que maiores pontuações foram relacionadas ao gênero feminino, raça branca, aposentados, tabagistas, etilistas, com HAS e DM. Ressalta-se melhor autoestima em aposentados/pensionistas, não obstante, foi observado o contrário em estudos que apresentaram associações positivas com a ocupação, uma vez que faz sentir-se útil, capaz, inserido num ciclo social e, consequentemente, apresente melhor julgamento de $\mathrm{si}^{(2,7,19,20)}$.

A literatura evidencia maior ocorrência de úlceras venosas em idosas, demonstrando que sua prevalência aumenta com a idade e que mulheres possuem maior probabilidade de desenvolvê-las, devido repercussões da gestação, distúrbios neuroendócrinos, presença de veias varicosas e insuficiência venosa crônica $\left.{ }^{(7,13,19}\right)$. Além disso, existe uma maior procura das mulheres pelo serviço de saúde quando comparado aos homens $^{(8,14)}$

Uma revisão que avaliou o impacto da úlcera venosa em diversas características psicoemocionais identificou depressão, baixa autoestima, auto aversão, isolamento social e baixa qualidade de vida como as de maior ocorrência ${ }^{(21)}$. Autores nacionais, ao avaliarem a intensidade de sintomas de depressão em pacientes com úlcera venosa, encontraram níveis variados de sintomas depressivos ${ }^{(2,4,15)}$.

Em outros estudos, as úlceras podem não ser apenas um dano físico, pois, para seu portador, podem ter diversos significados: algo que dói sem necessariamente ter estímulos sensoriais, uma marca, uma perda irreparável ou, até mesmo, uma doença incurável. Os fatores significantes para a satisfação de viver em relação aos problemas de saúde são o relacionamento social, o recurso financeiro e a autoestima ${ }^{(4,22,23)}$.

O instrumento SF-36 avalia, em seus domínios, questões, como capacidade funcional, limitação por aspecto físico, dor, estado geral de saúde, vitalidade, aspectos sociais, aspecto emocional e
Em outros estudos, as úlceras podem não ser apenas um dano físico, pois, para seu portador, podem ter diversos significados: algo que dói sem necessariamente ter estímulos sensoriais, uma marca, uma perda irreparável ou, até mesmo, uma doença incurável. Os fatores significantes para a satisfação de viver em relação aos problemas de saúde são o relacionamento social, o recurso financeiro e a autoestima. saúde mental. O domínio do SF-36 que apresentou mais comprometimento, neste estudo, foi Aspecto Físico, evidenciado em estudos prospectivos em pacientes portadores de úlceras traumáticas com a aplicação do questionário ${ }^{(4,8)}$.

Neste estudo, quando comparado o domínio do SF-36 Capacidade Funcional, são perceptíveis melhores resultados no gênero masculino, raça parda, não fumante, não etilista, com HAS e DM. Em relação ao Aspecto Físico e Emocional, melhores resultados ocorreram no gênero masculino, raça parda, tabagistas, não etilistas, com HAS e sem DM. Quando avaliados Dor e Estado Geral de Saúde são perceptíveis melhores resultados no gênero feminino, raça negra, tabagistas, etilistas, diferindo apenas na ocorrência de HAS e DM (para o domínio Estado Geral de Saúde foi relacionada a presença de HAS e DM, proporcionalmente oposto ao ocorrido para o domínio Dor). Analisando os domínios Vitalidade e Saúde Mental, as maiores pontuações ocorreram no gênero masculino, pardos, não fumantes, sem HAS e com DM. Já o Aspecto Social apresentou melhores resultados no gênero feminino, raça parda, tabagista, não etilista, sem HAS e com $\mathrm{DM}^{(4,8,24)}$.

Estudos internacionais indicam que a ocorrência de úlceras venosas aumenta com a idade. Alguns estudos anteriores no Brasil mostraram associação de úlceras venosas com baixa renda e escolaridade, lesões únicas e recorrentes e presença de processo de dor. No entanto, podemos ver poucos dados estatísticos sobre a prevalência desse tipo de úlcera no país e em diferentes regiões, bem como os fatores associados à história da ferida e seu tratamento, o que implica na subestimação do problema ${ }^{(9,24)}$.

O elevado número de lafaietenses com UV (1,64 à cada 1000 habitantes) aponta para a necessidade de ações de saúde para a assistência destes munícipes. Nessa perspectiva, a investigação sobre a QV desses pacientes pode corroborar na formulação de políticas públicas e auxiliar os profissionais na formulação 
de terapêutica proposta para o tratamento dos doentes.

\section{CONCLUSÕES}

O estudo possibilitou observar que houve maior comprometimento relacionado ao domínio Aspecto físico, enquanto Capacidade Funcional e Aspecto físico e emocional apresentaram resultados mais satisfatórios em homens, não fumantes e não etilistas. Nota-se que o domínio Aspecto Social apresenta resultados melhores em mulheres, de raça parda, tabagistas.

De um modo geral, percebe-se que o diagnóstico de úlcera venosa representa um impacto negativo na autoestima tanto de pessoas do gênero masculino, como
“

...percebe-se que o diagnóstico de úlcera venosa representa um impacto negativo na autoestima tanto de pessoas do gênero masculino, como feminino... feminino; porém os homens apresentam melhores escores relacionados à melhoria de qualidade de vida.

Contribuições para a área da enfermagem, saúde ou política

A principal contribuição deste estudo para o campo da pesquisa científica em úlceras venosas e para a enfermagem foi identificar quais são os fatores que afetam a qualidade de vida e capacidade funcional nos pacientes com úlceras venosas crônicas. A partir da caracterização desses fatores é possível realizar um plano assistencial para promoção e prevenção dos agravamentos. A realização do cuidado multidisciplinar permite alcançar maior adesão ao tratamento e a cicatrização de ulceras venosas em menor tempo possível para redução de custos financeiros.

\section{Referências}

1. Costa IKF, Dantas DV, Tibúrcio MP, Medeiros LP, Torres GV, Melo GSM. Protocolo de assistência a pessoas com úlcera venosa na Atenção Primária: revisão integrativa da literatura. Rev Online de Pesquisa. 2017;9(2):566-74.

2. Oliveira RA, Araújo RO, Costa VG, Silva LLA, Morais CCF, Torres SMSGSO, et al. Self-efficacy, self-esteem and adherence to treatment in people with venous ulcer in primary health care. Biosci J. 2017;33(6):1679-87.

3. Duffrayer KM, Joaquim FL, Camacho ACLF. Orientações em saúde: estratégia de promoção à capacidade funcional nas úlceras venosas. Rev Enferm UFPE. 2018;12(7):1901-11.

4. Joaquim FL, Silva RMCRA, Garcia-Caro MP, Cruz-Quintana F, Pereira ER. Impacto das úlceras venosas na qualidade de vida dos pacientes: revisão integrativa. Rev Bras Enferm. 2018;71(4):2137-46.

5. Norman G, Westby MJ, Rithalia AD, Stubbs N, Soares MO, Dumville JC. Dressings and topical agents for treating venous leg ulcers. Cochrane Database Syst Rev. 2018(6):1-293.

6. Torres SMSSO, Araújo RO, Costa IKF, Tibúrcio MP, Sousa AJG, Pergola-Marconato $A M$, et al. Health-related quality of life in patients with venous leg ulcer treated in primary care in Brazil and Portugal. PLoS. 2018;13(4):e0195990.

7. Silva PAS, Souza NVDO, Santos DM, Oliveira EB, Souza MB, Nascimento DC. Homens com úlcera venosa de perna e as implicações para vida laboral. Rev Enferm UERJ. 2019a;27:e40876.

8. Couto RC, Leal FJ, Pitta GBB, Andreoni S. Responsividade do questionário de qualidade de vida CCVUQ-Br em portadores de úlcera venosa crônica. J Vasc Bras. 2020;19:1-9.

9. Oliveira SB, Soares DA, Pires PS. Prevalence of venous ulcers and associated factors among adults of a health center in Vitória da Conquista - BAJ. J Fundam Care. 2015;7(3):2659-69.

10. Ziaja D, Sznapka M, Grzela J, Kostecki J, Biolik G, Pawlicki K, et al. Regional variations of symptoms of the chronic venous disease among primary health care patients in Poland. Acta Angiol. 2015;21(2):31-9.

11. Assunção IKFC, Medeiros LP, Dias TYAF, Salvetti MG, Dantas DV, Torres GV. Protocol validation for people with venous ulcers: a quantitative study. Online Braz J Nurs. 2016;15(2):226-35.

12. Harding K. Challenging passivity in venous leg ulcer care - the $A B C$ model of management. Int Wound J. 2016;13(6):1378-4.
13. Silva MH, Jesus MCP, Tavares RE, Caldeira EAC, Oliveira DM, Merighi MAB. Experiência de pessoas adultas e idosas frente à adesão aos cuidados com a úlcera varicosa. Rev Gaúcha Enferm. 2019b;40:1-8.

14. Finlayson K, Miaskowski C, Alexander K, Liu WH, Aouizerat B, Parker C, $\mathrm{PhD}$, et al. Distinct Wound Healing and Quality-of-Life outcomes in subgroups of patients with venous leg ulcers with different symptom cluster experiences. J Pain Symplom Manege. 2017;53(5):871-9.

15. Joaquim FL, Camacho ACLF, Silva RMCRA, Leite BS, Queiroz RS, Assis CRC. Repercussão da visita domiciliar na capacidade funcional de pacientes com úlceras venosas. Rev Bras Enferm. 2017;70 (2):304-11.

16. Borges EL, Nascimento-Filho HM, Pires-Júnior JF. Prevalência de Lesões Crônicas de Município de Zona da Mata Mineira. Rev Min Enferm. 2018;22:e1143.

17. Laguardia J, Campos MR, Travassos C, Najar AL, Anjos LA, Vasconcellos MM. Dados normativos brasileiros do questionário Short Form-36 versão 2. Rev Bras Epidemiol. 2013;16(4): 889-97.

18. Salvetti MG, Costa IK, Dantas DV, Freitas CC, Vasconcelos QL e Torres GV. Prevalence of pain and associated factors in venous ulcer patients. Rev Dor. 2014;15(1):17-20.

19. Silva DC, Budó MLD, Schimith MD, Ecco L, Costa IKF, Torres GV Experiences constructed in the process of living with a venous ulcer. Cogitare Enferm. 2015;20(1):13-9.

20. Souza AJG, Campos SMDL, Salvetti MG, Queiroz CG, Torres GV, Costa IKF. Autoestima de pessoas com úlcera venosa. Rev Rene. 2017;18(5):569-76.

21. Maddox D. Effects of venous leg ulceration on patients' quality of life. Nurs Standard. 2012;26(38):42-9.

22. Fagerström C, Borg C, Balducci C, Burbolt V, Wenger CG, Ferring D, et al. Life satisfaction and associated factor's among people aged 60 years and above in six European countries. Res Qual Life. 2007;2:33-50.

23. Lucas LS, Martins JT, Robazzi ML. Qualidade de vida dos portadores de ferida em membros inferiores - úlcera de perna. Cienc Enferm. 2008;14(1):43-52. 24. Couto RC, Leal FJ, Pitta GBB. Validação do questionário de qualidade de vida na úlcera venosa crônica em língua portuguesa (Charing Cross Venous Ulcer Questionnaire - CCVUQ-Brasil). J Vasc Bras. 2016;15(1):4-10. 